\title{
THERMAL DIFFUSION AND CHEMICAL REACTION EFFECTS ON UNSTEADY FLOW PAST A VERTICAL POROUS PLATE WITH HEAT SOURCE DEPENDENT IN SLIP FLOW REGIME
}

\author{
Siva Kumar Narasu' ${ }^{1}$ B. Rushi Kumar ${ }^{*}$, A. G. Vijaya Kumar ${ }^{3}$
}

${ }^{1,2,3}$ Fluid Dynamics Division, School of Advanced Sciences, VIT University, Vellore, T.N, INDIA - 632014, "Email: rushibkumar@gmail.com

\begin{abstract}
:
The present study investigates an analytical solution of free convective unsteady fluid flow in the presence of thermal diffusion and chemical reaction past a vertical porous plate with heat source dependent in slip flow regime. The plate is assumed to move with a constant velocity in the direction of fluid flow while free stream velocity is assumed to follow exponentially increasing small perturbation law. The velocity, temperature, and concentration profiles are presented graphically for different values of the parameters entering into the problem. Finally, the effects of pertinent parameters on the skin friction coefficient, Nusselt number, and Sherwood numbers are studied and are shown through graphs and tables by using perturbation technique. It is observed in the case of air that the temperature decreases very rapidly whereas, in the case of liquid, the temperature decreases steadily.
\end{abstract}

Keywords:Free convection, heat and mass transfer, slip flow regime, thermal diffusion, heat source, chemical reaction.

\author{
NOMENCLATURE \\ $u^{*}$ The component of velocity along $\mathrm{x}$-axis $(\mathrm{m} / \mathrm{s})$ \\ $v^{*}$ The component of velocity along y-axis $(\mathrm{m} / \mathrm{s})$ \\ $t$ Time $(\mathrm{S})$ \\ $\mathrm{g}$ The acceleration due to gravity $\left(\mathrm{ms}^{-2}\right)$ \\ $\beta_{\mathrm{T}}$ Thermal expansion coefficient \\ $\beta_{\mathbf{c}}$ Concentration expansion coefficient \\ U Kinematic viscosity $\left(\mathrm{m}^{2} \mathrm{~s}^{-1}\right)$ \\ $k^{*}$ Permeability of the porous medium non- \\ dimensional form \\ $S_{0}$ Soret effect \\ $\rho$ Density of the dusty fluid $\left(\mathrm{kgm}^{-3}\right)$ \\ $Q$ Heat source \\ $C_{p}$ Specific heat at constant pressure (kj/kg.k) \\ $T^{*}$ Temperature in non- dimensional form \\ $C^{*}$ Concentration in non- dimensional form \\ $U_{0}$ Suction velocity $(\mathrm{m} / \mathrm{s})$
}

$D$ Molecular diffusivity $\left(\mathrm{m}^{2} / \mathrm{s}\right)$

$D_{l}$ Thermal diffusivity $\left(\mathrm{m}^{2} / \mathrm{s}\right)$

$K_{c}^{*}$ Chemical reaction parameter in non- dimension form

$G_{T}$ Thermal Grashof number

$G_{M} \quad$ Mass Grashof number

$K_{c} \quad$ Chemical reaction parameter

$K$ Permeability of porous medium $\left(\mathrm{m}^{2}\right)$

Pr Prandtl number

$\boldsymbol{H}$ Heat source parameter $\left(\mathrm{w} / \mathrm{m}^{2} \mathrm{~K}\right)$

Sc Schmidt number

$M_{x}$ Maxwell's reflection coefficient

$T_{\infty}^{*}$ Temperature of the fluid far away from the plate

$C_{\infty}^{*}$ Concentration of the fluid far away from the plate

$T_{w}^{*}$ Temperature of the fluid at the plate

$C_{w}^{*}$ Concentration of the fluid at the plate

\section{Introduction}

The problem of free convection flow involving the combined mechanism of heat and mass transfer are encountered in many natural processes, in many chemical processing systems, and in many industrial applications. The study of free convective heat and mass transfer flow has become the object of extensive research as the effects of heat transfer along with mass transfer are dominant features in many engineering 
applications such as cooling of nuclear reactors, rocket nozzles, high-speedaircraft and their atmospheric reentry, chemical devices process equipment.

The phenomenon of free convection arises in the fluid when the temperature changes cause density variation leading to buoyancy forces acting on the fluid elements. This can be seen in our everyday life in the atmospheric flow, which is driven by temperature differences. There are many transport process occurring in nature due to temperature and chemical differences. The process of heat and mass transfer is encountered in aeronautics, fluid fuel nuclear reactor, chemical process industries and many engineering applications in which the fluid is a working medium and Chemical reaction can be codified either heterogeneous or homogeneous processes. Its effect depends on the nature of the reaction whether the reaction is heterogeneous or homogeneous. A reaction is of the firstorder if the rate of reaction is directly proportional to concentration itself. In nature, the presence of pure air or water is not possible. Some foreign mass may be present naturally mixed with air or water. The presence of a foreign mass in air or water causes some kind of chemical reaction. The study of such type of chemical reaction processes is useful for improving a number of chemical technologies, such as food processing, polymer production, manufacturing of ceramics and glassware.

Several authors have analyzed physical problems in this field. Chambre and Young (1958)analyzed the effect of homogeneous first order chemical reactions in the neighborhood of a flat plate for destructive and generative reactions. Das et al. (1994) studied the effect of first order reaction on the flow past an impulsively started infinite vertical plate with uniform heat flux and mass transfer. Alam and Rahman (2005) analyzed Dufour and Soret effects on MHD free convective heat and mass transfer flow past a vertical porous plate embedded in a porous medium.Anjali Devi and Kandasamy (2000) investigated the effect of chemical reaction on the flow in the presence of heat transfer and magnetic field. Muthucumaraswamy and Ganesan (2001) studied the effect of chemical reaction on flow past an impulsively started vertical plate with uniform heat and mass flux. A study on steady laminar free convection flow of an electrically conducting fluid along a porous vertical plate in the presence of heat source was carried out by Sharma and Mathur (1995). The effect of thermal diffusion on steady laminar free convective flow along a moving porous hot vertical plate in the presence of heat source with the mass transfer was studied by Varshney and Kumar (2004). Heat transfer in MHD free convection flow over an infinite vertical plate with time-dependent suction was investigated in detail by Mishra (2005). Sharma and Singh (2008) examined unsteady MHD free convective flow and heat transfer along a vertical porous plate with variable suction and internal heat generation.

In many practical applications, the particle adjacent to a solid surface no longer takes the velocity of the surface. The particle at the surface has a finite tangential velocity; it slips along the surface. The flow regime is called the slip flow regime and this effect cannot be neglected. Using these assumptions, Sharma and Chaudary (2003) discussed the free convection flow past a vertical plate in the slip-flow regime and also discussed its various applications for engineering purpose. Also, Sharma (2005) investigated the effect of periodic heat and mass transfer on the unsteady free convection flow past a vertical flat plate in the slip-flow regime when suction velocity oscillates in time. Coupled non-linear partial differential equations governing free convection flow, heat, and mass transfer have been obtained analytically using the perturbation technique. The fluids considered in this investigation are air $\left(P_{r}=0.71\right)$ and water $\left(P_{r}=7\right)$ in the presence of Hydrogen $\left(S_{c}=0.22\right)$. Magnetohydrodynamic convective heat and mass transfer in a boundary layer slip flow past a vertical permeable plate with thermal radiation and chemical reaction were investigated by Pal and Talukdar (2010). Kumar and Gangadhar (2012) have studied the heat generation effects on MHD boundary layer flow of a moving vertical plate with suction. Das and Mitra (2009) discussed the unsteady mixed convective MHD flow and mass transfer past an accelerated infinite vertical plate with suction. Recently, Das et al. (2009) analyzed the effect of mass transfer on MHD flow and heat transfer past a vertical porous plate through a porous medium under oscillatory suction and heat source. Das et al. (2007) investigated numerically the unsteady free convective MHD flow past an accelerated vertical plate with suction and heat flux.Dufour and Soret effects on steady MHD free convection and transfer fluid flow through a porous medium was analyzed by Mahmud and Nazmul (2007). Das et al. (2006) estimated the mass transfer effects on unsteady flow past an accelerated vertical porous plate with suction employing finite difference analysis. Kumar et al.(2009) investigated effects of chemical reaction and mass transfer on MHD unsteady free convection flow past an infinite vertical plate with constant suction and 
heat sink. Kumar et al. (2015), have examined the effects of thermal diffusion and radiation effects on unsteady free convection flow in the presence of magnetic field fixed relative to the fluid or to the plate. Hayat et al. (2010) analyzed a mathematical model in order to study the heat and mass transfer characteristics in mixed convection boundary layer flow over a linearly stretching vertical surface in a porous medium filled with a viscous - elastic fluid, by taking into account the diffusion thermal and thermal diffusion effects. Recently, Poonia and Chaudhary (2010) studied an unsteady, two-dimensional, hydro-magnetic, laminar mixed convective boundary layer flow of an incompressible and electrically conducting fluid along an infinite vertical plate embedded in the porous medium with heat and mass transfer, by taking into account the effects of viscous dissipation.In further studies by Saxena et al. (2009) investigates the unsteady two-dimensional magnetohydrodynamic heat and mass transfer free convection flow of an incompressible, viscous, electrically conducting polar fluid through a porous medium past a semi-infinite vertical porous moving plate in the presence of a transverse magnetic field with thermal diffusion and heat generation. The plate moves with a constant velocity in the longitudinal direction, and the free stream velocity follows an exponentially increasing or decreasing small perturbation law. Kumar (2013) analyzed the MHD boundary layer flow of heat and mass transfer over a stretching sheet with slip effect.

The objective of the present paper is to study an analytical solution of free convective thermal diffusion and chemical reaction effects on unsteady flow past a vertical porous plate with heat source dependent in slip flow regime. In obtaining the solution, the heat source term is taken into account in the energy equation and chemical reaction parameter, thermal diffusion parameter is taken into account in the concentration equation. To the best of author's knowledge, this model has not been discussed in the literature. The Permeability of the porous medium and suction velocity are considered to be as exponentially decreasing the function of time.

\section{Mathematical Formulation}

We consider a two-dimensional unsteady free convection flow of an incompressible viscous fluid past an infinite vertical porous plate. In rectangular Cartesian coordinate system, we take $\mathrm{x}$-axis along the plate in the direction of flow and y-axis normal to it. Further, the flow is considered in the presence of thermal diffusion and chemical reaction effect past a vertical porous plate with heat source dependent in slip flow regime. In the analysis for small velocity the viscous dissipation and Darcy's dissipation are neglected. It is assumed that radioactive heat transfer is negligible in the energy equation, the fluid has small electric conductivity and the electromagnetic force is very small.

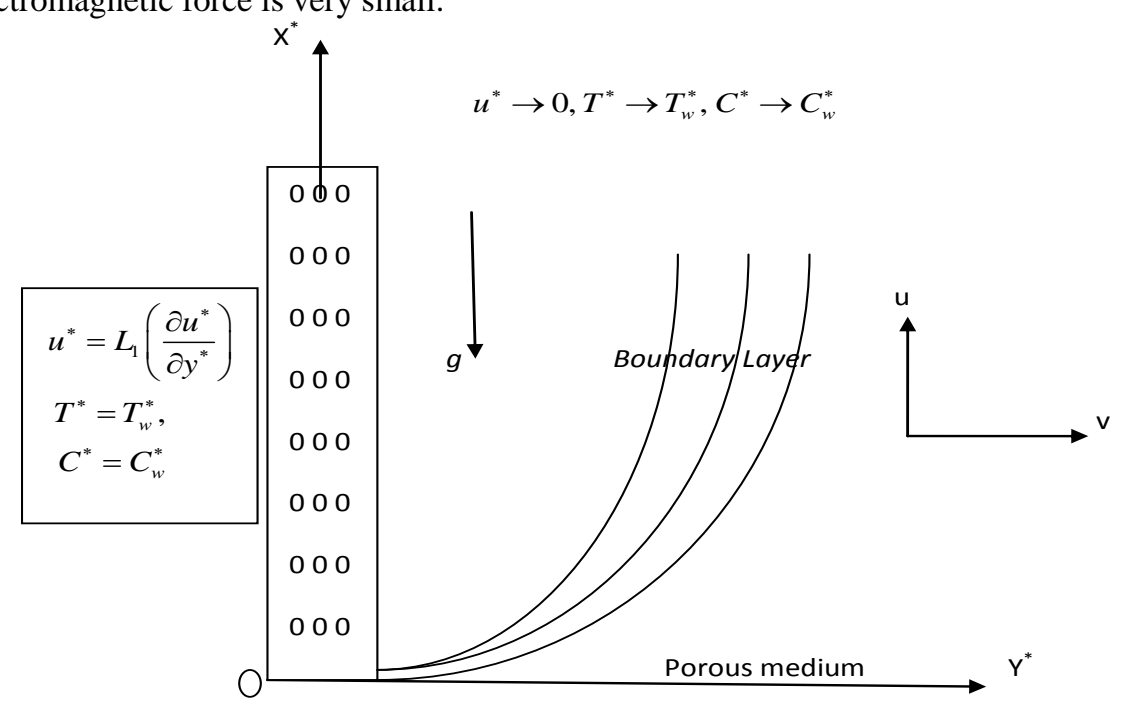

Fig. A: Physical model and coordinate system 
The flow in the medium is entirely due to buoyancy force caused by temperature difference between the porous plate and the fluid. Under the above assumptions, the conservation of mass, momentum, energy, concentration is given bythe following relations, respectively (Rao et al., 2013).

$$
\begin{aligned}
& \frac{\partial v^{*}}{\partial y^{*}}=0 \\
& \frac{\partial u^{*}}{\partial t^{*}}+v^{*} \frac{\partial u^{*}}{\partial y^{*}}=g \beta_{T}\left(T^{*}-T_{\infty}^{*}\right)+g \beta_{C}\left(C^{*}-C_{\infty}^{*}\right)+v \frac{\partial^{2} u^{*}}{\partial y^{*^{2}}}-\frac{v}{k^{*}(t)} u^{*} \\
& \frac{\partial T^{*}}{\partial t^{*}}+v^{*} \frac{\partial T^{*}}{\partial y^{*}}=k_{T} \frac{\partial^{2} T^{*}}{\partial y^{*^{2}}}+\frac{Q^{*}}{\rho c_{p}} \frac{\partial T}{\partial y} \\
& \frac{\partial C^{*}}{\partial t^{*}}+v^{*} \frac{\partial C^{*}}{\partial y^{*}}=D \frac{\partial^{2} C^{*}}{\partial y^{*^{2}}}-k_{c}^{*}\left(C^{*}-C_{\infty}^{*}\right)+D_{1} \frac{\partial^{2} T^{*}}{\partial y^{*^{2}}}
\end{aligned}
$$

The boundary conditions relevant to the problem are

$$
\begin{aligned}
& u^{*}=L_{1}\left(\frac{\partial u^{*}}{\partial y^{*}}\right), T^{*}=T_{w}^{*}, C^{*}=C_{w}^{*} \text { at } y^{*}=0 \\
& u^{*} \rightarrow 0, T^{*} \rightarrow T_{w}^{*}, C^{*} \rightarrow C_{w}^{*} \text { as } y^{*} \rightarrow \infty \\
& \text { Where } L_{1}=\frac{2-M_{x}}{M_{x}}
\end{aligned}
$$

The equation of continuity (1) yields that $\mathrm{v}^{*}$ is either a constant or some function of time, hence we assume that

$$
v^{*}=-U_{0}\left(1+\varepsilon e^{-n^{*} t^{*}}\right)
$$

The negative sign indicates that the suction velocity acts towards the plate.

The Permeability of the porous medium in non-dimensional form is considered as

$$
k^{*}(t)=k_{0}^{*}\left(1+\varepsilon e^{-n^{*} t^{*}}\right)
$$

Now, we introduce the following non-dimensional quantities

$$
u=\frac{u^{*}}{U_{0}}, y=\frac{y^{*} U_{0}}{v}, n=\frac{4 v n^{*}}{U_{0}^{2}}, \mathrm{t}=\frac{U_{0}^{2} t^{*}}{4 v}, T=\frac{T^{*}-T_{\infty}^{*}}{T_{w}^{*}-T_{\infty}^{*}}, K=\frac{k_{0}^{*^{2}} U_{0}^{2}}{v^{2}}, C=\frac{C^{*}-C_{\infty}^{*}}{C_{w}^{*}-C_{\infty}^{*}}
$$

Introducing the equations (6), (7) in the equations (2), (3), (4), we obtain

$$
\begin{aligned}
& \frac{1}{4} \frac{\partial u}{\partial t}-\left(1+\varepsilon e^{-n t}\right) \frac{\partial u}{\partial y}=G_{T} T+G_{M} C+\frac{\partial^{2} u}{\partial y^{2}}-M_{1} u \\
& \frac{1}{4} \frac{\partial T}{\partial t}-\left(1+\varepsilon e^{-n t}\right) \frac{\partial T}{\partial y}=\frac{1}{P_{r}} \frac{\partial^{2} T}{\partial t^{2}}+H \frac{\partial T}{\partial y} \\
& \frac{1}{4} \frac{\partial C}{\partial t}-\left(1+\varepsilon e^{-n t}\right) \frac{\partial C}{\partial y}=\frac{1}{S_{c}} \frac{\partial^{2} C}{\partial t^{2}}-k_{c} C+S_{0} \frac{\partial^{2} T}{\partial y^{2}}
\end{aligned}
$$


and the boundary conditions (5) reduce to

$$
\begin{aligned}
& u=h\left(\frac{\partial u}{\partial y}\right), T=1, C=1 \text { at } y=0 \\
& u \rightarrow 0, T \rightarrow 0, C \rightarrow 0 \text { as } y \rightarrow \infty
\end{aligned}
$$

Where

$$
\begin{aligned}
& h=\frac{L U_{0}^{2}}{v}, G_{T}=\left(\frac{g \beta_{T}\left(T_{w}^{*}-T_{\infty}^{*}\right) v}{U_{0}^{3}}\right) T, G_{M}=\left(\frac{g \beta_{c}\left(\mathrm{C}_{w}^{*}-C_{\infty}^{*}\right) v}{U_{0}^{3}}\right) C, M_{1}=\left(\frac{1}{\mathrm{~K}\left(1+\varepsilon e^{-n t}\right)}\right) u \\
& P_{r}=\frac{\rho c_{p} v}{k_{T}}, H=\frac{Q^{*} v}{\rho c_{p} U_{0}^{2}\left(T_{w}^{*}-T_{\infty}^{*}\right)}, S_{c}=\frac{v}{D}, k_{c}=\frac{k_{c}^{*} v}{U_{0}^{2}}, S_{0}=\frac{D_{1}\left(T_{w}^{*}-T_{\infty}^{*}\right)}{\left(\mathrm{C}_{w}^{*}-C_{\infty}^{*}\right) v}
\end{aligned}
$$

\section{Solution of the Problem}

In order to solve the nonlinear partial differential equations, the above systems of partial differential equations (8), (9) and (10) are reduced to a system of ordinary differential equations in a dimensionless form. To obtain the solution, the following perturbation method, which is given by Singh et al. (2006), is used for ' $\varepsilon$ ' $<<1$. The velocity, temperature and concentration are assumed in the following form:

$$
\begin{aligned}
& \mathrm{u}(y, t)=f_{0}(y)+\varepsilon f_{1}(y) \mathrm{e}^{-n t} \\
& \mathrm{~T}(y, t)=g_{0}(y)+\varepsilon g_{1}(y) \mathrm{e}^{-n t} \\
& \mathrm{C}(y, t)=h_{0}(y)+\varepsilon h_{1}(y) \mathrm{e}^{-n t}
\end{aligned}
$$

Substituting above expressions (12) in to the equations (8), (9), (10) and equating the coefficient of $\varepsilon^{0}, \varepsilon^{1}$ (neglecting $\varepsilon^{2}$ terms etc.,), we obtain the following set of ordinary differential equations

$$
\begin{aligned}
& f_{0}^{\prime \prime}+f_{0}^{\prime}-M_{1} f_{0}=-G_{T} g_{0}-G_{M} h_{0} \\
& f_{1}^{\prime \prime}+f_{1}^{\prime}+M_{2} f_{1}=-G_{T} g_{1}-G_{M} h_{1}-f_{0}^{\prime} \\
& g_{0}^{\prime \prime}+P_{r}(1+H) g_{0}^{\prime}=0 \\
& g_{1}^{\prime \prime}+P_{r}(1+H) g_{1}^{\prime}+\frac{n P_{r}}{4} g_{1}=-P_{r} g_{0}^{\prime} \\
& h_{0}^{\prime \prime}+S_{c} h_{0}^{\prime}-k_{c} S_{c} h_{0}=-S_{c} S_{0} g_{0}^{\prime \prime} \\
& h_{1}^{\prime \prime}+S_{c} h_{1}^{\prime}+S_{c}\left(\frac{n}{4}-k_{c}\right) h_{1}=S_{c} S_{0} g_{1}^{\prime \prime}-S_{c} h_{0}^{\prime}
\end{aligned}
$$

Here $M_{2}=\frac{n}{4}-M_{1}$

And the boundary conditions (11) reduce to

$$
\begin{aligned}
& f_{0}=h f_{0}^{\prime}, f_{1}=h f_{1}^{\prime}, g_{0}=1, g_{1}=0, h_{0}=1, h_{1}=0 \text { at } y=0 \\
& f_{0} \rightarrow 0, f_{1} \rightarrow 0, g_{0} \rightarrow 0, g_{1} \rightarrow 0, h_{0} \rightarrow 0, h_{1} \rightarrow 0 \text { as } y \rightarrow \infty
\end{aligned}
$$


The equations from (13) to (18) are $2^{\text {nd }}$ order linear differential equations with constant coefficients. The solutions of these paired equations under the corresponding boundary conditions (19) are

$$
\begin{aligned}
& g_{o}=e^{-m_{1} y} \\
& g_{1}=A_{1}\left(e^{-m_{1} y}-e^{-m_{2} y}\right) \\
& h_{0}=e^{-m_{3} y}+A_{2}\left(e^{-m_{1} y}-e^{-m_{3} y}\right) \\
& h_{1}=\left(-A_{3}-A_{4}-A_{5}\right) e^{-m_{4} y}+A_{3} e^{-m_{1} y}+A_{4} e^{-m_{2} y}+A_{5} e^{-m_{3} y} \\
& f_{0}=A_{8} e^{-m_{5} y}-A_{6} e^{-m_{1} y}-A_{7} e^{-m_{3} y} \\
& f_{1}=A_{14} e^{-m_{6} y}+A_{9} e^{-m_{1} y}+A_{10} e^{-m_{2} y}+A_{11} e^{-m_{3} y}+A_{12} e^{-m_{4} y}+A_{13} e^{-m_{5} y}
\end{aligned}
$$

Introducing the equations (20) to (25) in the equations (12), we get the expressions for velocity, temperature and concentration as

$$
\begin{aligned}
\mathrm{u}(\mathrm{y}, \mathrm{t})= & \left(A_{8} e^{-m_{5} y}-A_{6} e^{-m_{1} y}-A_{7} e^{-m_{3} y}\right) \\
& +\varepsilon\left(A_{14} e^{-m_{6} y}+A_{9} e^{-m_{1} y}+A_{10} e^{-m_{2} y}+A_{11} e^{-m_{3} y}+A_{12} e^{-m_{4} y}+A_{13} e^{-m_{5} y}\right) e^{-n t} \\
\mathrm{~T}(y, t)= & \left(e^{-m_{1} y}\right)+\varepsilon\left(A_{1}\left(e^{-m_{1} y}-e^{-m_{2} y}\right)\right) e^{-n t} \\
\mathrm{C}(y, t)= & \left(e^{-m_{3} y}+A_{2}\left(e^{-m_{1} y}-e^{-m_{3} y}\right)\right) \\
& +\varepsilon\left(\left(-A_{3}-A_{4}-A_{5}\right) e^{-m_{4} y}+A_{3} e^{-m_{1} y}+A_{4} e^{-m_{2} y}+A_{5} e^{-m_{3} y}\right) e^{-n t}
\end{aligned}
$$

\section{Skin-friction:}

The expression for the skin-friction $(\tau)$ at the plate is

$$
\tau=\left(\frac{\partial u}{\partial y}\right)_{y=o}=\left(\frac{\partial f_{0}}{\partial y}\right)_{y=o}+\varepsilon\left(\frac{\partial f_{1}}{\partial y}\right)_{y=o} e^{-n t}=A_{15}+\varepsilon A_{16} e^{-n t}
$$

\section{Nusselt number:}

The expression for nusselt number $\left(N_{u}\right)$ in terms of the rate of heat transfer is

$$
N_{u}=\left(\frac{\partial T}{\partial y}\right)_{y=o}=\left(\frac{\partial g_{0}}{\partial y}\right)_{y=o}+\varepsilon\left(\frac{\partial g_{1}}{\partial y}\right)_{y=o} e^{-n t}=-m_{1}+\varepsilon A_{17} e^{-n t}
$$

\section{Sherwood number:}

The expression for Sherwood number in terms of the rate of mass transfer is

$$
S_{h}=\left(\frac{\partial C}{\partial y}\right)_{y=o}=\left(\frac{\partial h_{0}}{\partial y}\right)_{y=o}+\varepsilon\left(\frac{\partial h_{1}}{\partial y}\right)_{y=o} e^{-n t}=A_{18}+\varepsilon A_{19} e^{-n t}
$$

\section{Results and Discussion}

The problem of thermal diffusion and chemical reaction effects on unsteady fluid flow past a vertical porous plate with heat source dependent in slip flow regime has been investigated and the analytical solution for velocity, temperature and concentration have been presented in the previous section. The skin friction 
coefficient, heat transfer coefficient, and mass transfer coefficient are also found. From the available analytical solutions, the numerical values for the distributions of velocity, temperature, concentration, skin friction coefficient, Nusselt number and Sherwood number are calculated by fixing various values of the nondimensional parameters involved in the problem. In the present work, we have chosen $t=1, \varepsilon=0.1, K=1$ and $n=1$ while the other non-dimensional parameters take various values.

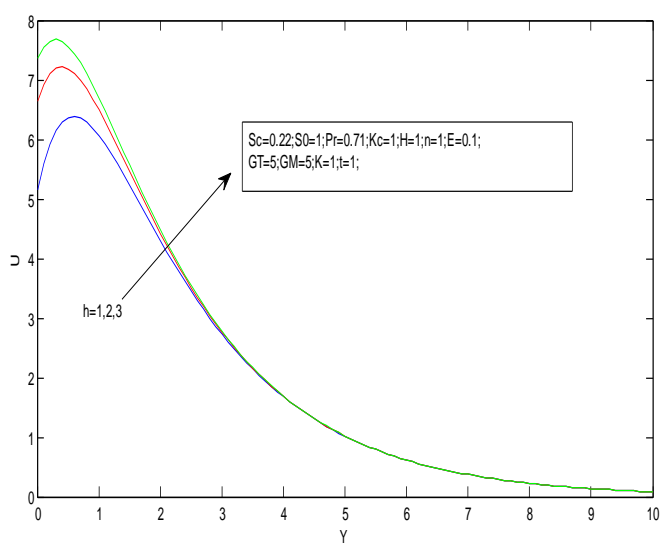

Fig. 1: The effect of slip parameter $h$ on velocity

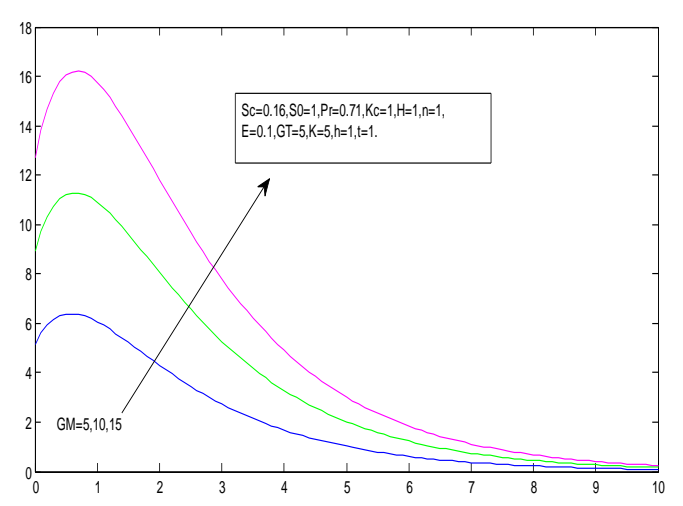

Fig. 3: The effect of Mass Grashoff number $G_{M}$ on velocity

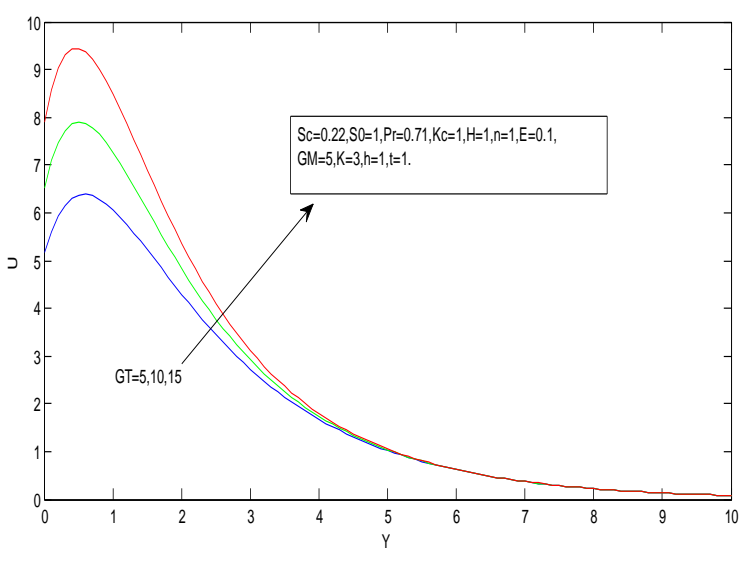

Fig. 2: The effect of Thermal Grashoff number $G_{T}$ on velocity

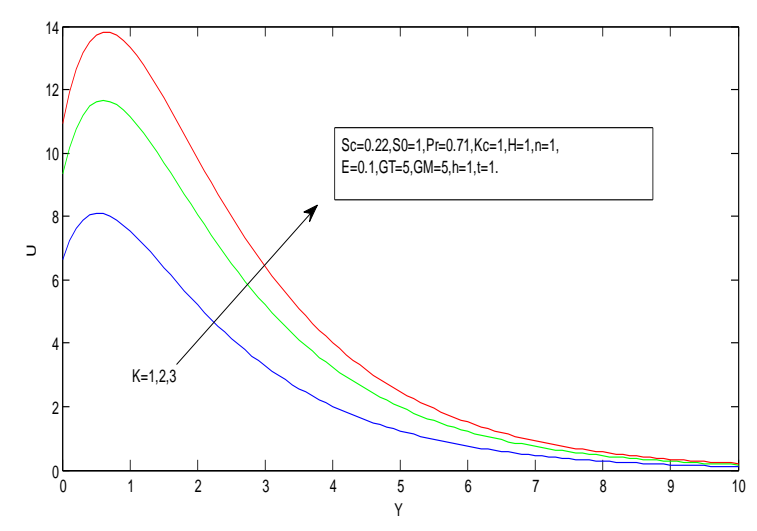

Fig. 4: The effect of porous medium $K$ on velocity

To assess the physical depth of the problem, the effects of various parameters like slip parameter $h$, Thermal Grashof number $G_{T}$, Permeability of Porous medium $K$, Heat source parameter $H$, Chemical reaction parameter $K c$, Modified Grashof number $G_{M}$, Schmidt number $S_{c}$, Soret number $S_{0}$ and Prandtl Number $P_{r}$ on velocity distribution, temperature distribution, and concentration distribution are studied in Figs. (1) to (9), while keeping the other parameters as constants. Fig. (1) depicts the velocity profiles with the variations in $h$, it is observed that the significance of the velocity is high near the plate and thereafter it decreases and reaches to the stationary position at the other side of the plate. As expected, velocity increases with an increase in $h$. The effects of $G_{T}$ on velocity distribution are presented in Fig. (2). From this figure, it is noticed that velocity increases as an increase in $G_{T}$. In Fig. (3) the effects of $G_{M}$ on velocity are shown. From this figure, it is noticed that velocity increases as $G_{M}$ increases. From Fig. (4), it is observed that the velocity increases as permeability of porous medium $K$ increases. In Figs. (5) \& (6) the temperature distribution increases as heat source parameter HandPrandtl Number $P_{r}$ decreases respectively. In Fig. (7), concentration increases with an increase in $S_{0}$. In Figs. (8) and (9) the concentration decreases as $S_{c}$ and $K_{c}$ increase respectively. To be realistic, the numerical values of Prandtl 
number $P_{r}$ are chosen as $P_{r}=0.71$ and $P_{r}=7$, which corresponds to air and water at $20^{\circ} \mathrm{C}$ respectively.In heat transfer problems, the Prandtl number controls the relative thickness of the momentum and thermal boundary layers. When Pr is small, it means that the heat diffuses quickly compared to the velocity. This means that for liquid metals the thickness of the thermal boundary layer is much bigger than the velocity boundary layer. The numerical values of the remaining parameters are chosen arbitrarily.

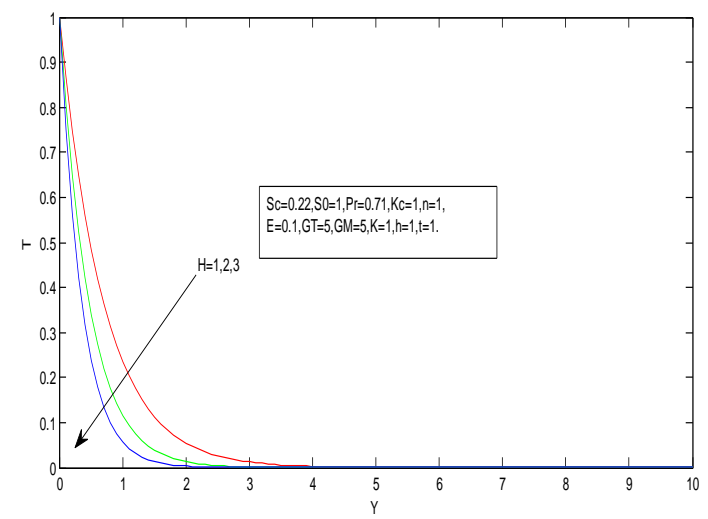

Fig. 5: The effects of Heat Source parameter $H$ on Temperature

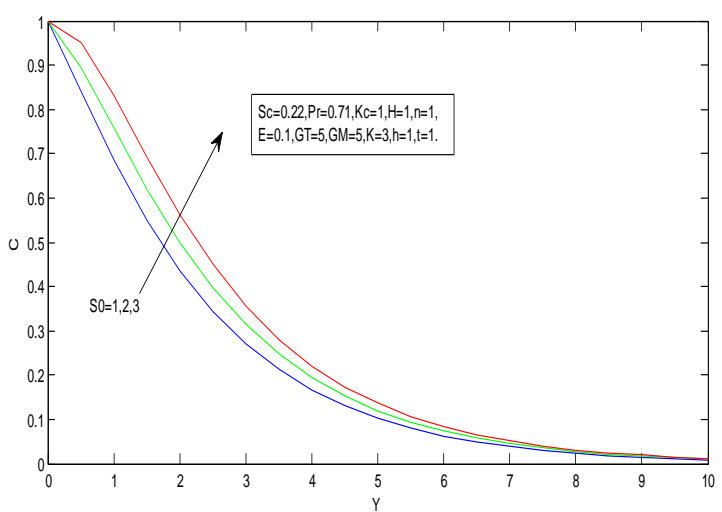

Fig. 7: The effects of $S_{0}$ on Concentration

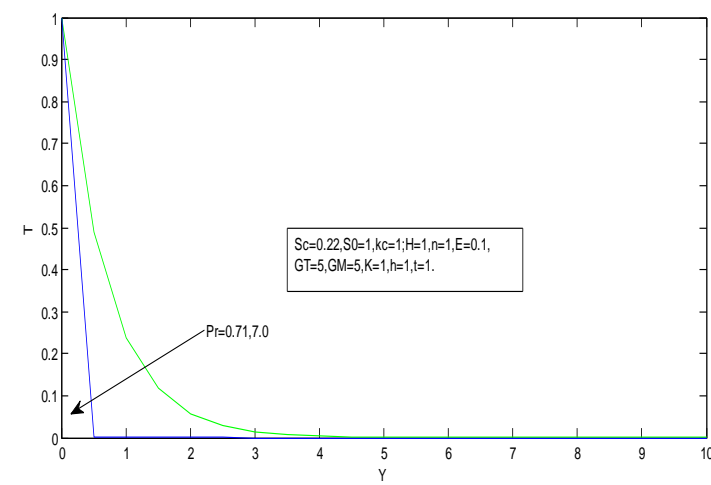

Fig. 6: The effects of Prandtl number $P_{r}$ on Temperature

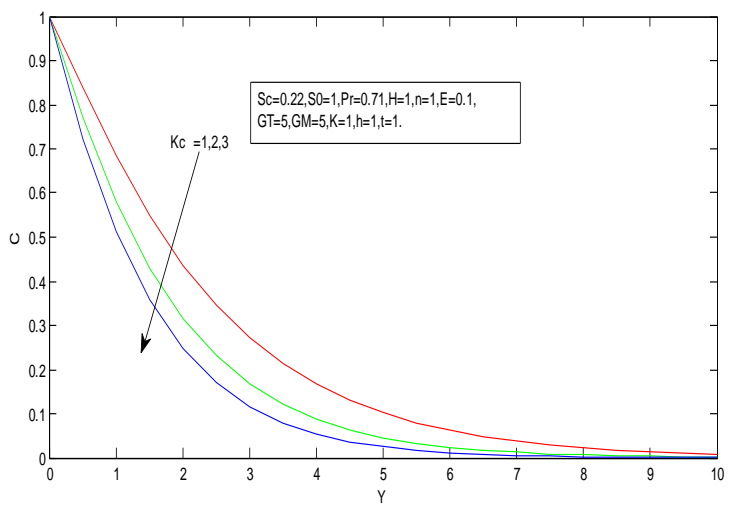

Fig. 8: The effects of Chemical reaction parameter $K_{c}$ on concentration

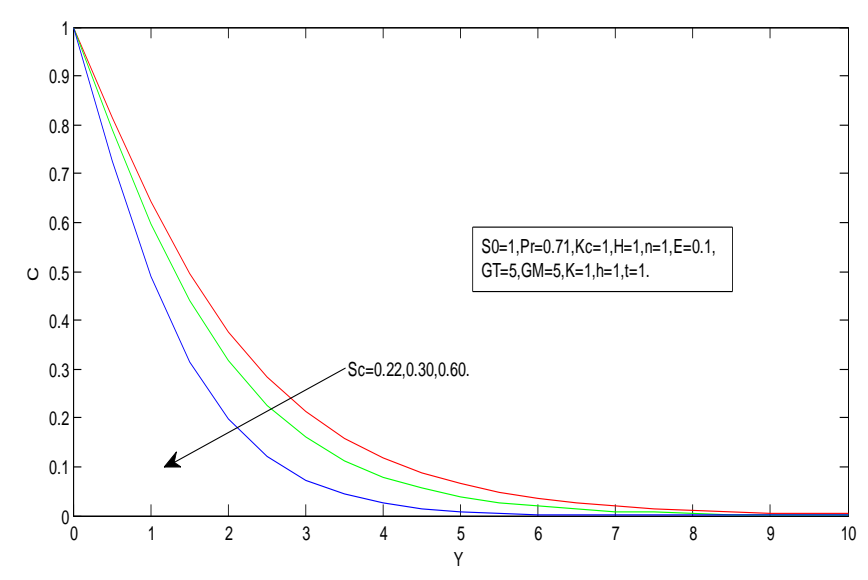

Fig. 9: The effects of Schmidt number $S_{c}$ on concentration 
The variations in skin friction, Nusselt number, and Sherwood number are studied at $n=1, t=1$ and $\varepsilon=0.1$ through the Tables (1) to (3).

Table 1: Skin friction at the plate when $P_{r}=0.71, n=1, t=1$ and $\varepsilon=0.1$

\begin{tabular}{|c|c|c|l|l|}
\hline \multicolumn{1}{|l|}{$\boldsymbol{G}_{\boldsymbol{T}}$} & $\boldsymbol{G}_{\boldsymbol{M}}$ & $\boldsymbol{H}$ & $\boldsymbol{K}$ & $\boldsymbol{\tau}$ \\
\hline 5 & 5 & 1 & 1 & 3.1537 \\
\hline 10 & 5 & 1 & 1 & 1.0803 \\
\hline 15 & 5 & 1 & 1 & 5.0068 \\
\hline 5 & 10 & 1 & 1 & 5.3809 \\
\hline 5 & 15 & 1 & 1 & 7.6080 \\
\hline 5 & 5 & 3 & 1 & 1.4073 \\
\hline 5 & 5 & 1 & 2 & 4.3995 \\
\hline 5 & 5 & 1 & 3 & 5.1290 \\
\hline
\end{tabular}

Table 2: Rate of heat transfer at the plate

\begin{tabular}{|c|c|c|}
\hline $\boldsymbol{P}_{\boldsymbol{r}}$ & $\boldsymbol{H}$ & $\boldsymbol{N}_{\boldsymbol{u}}$ \\
\hline 0.71 & 1 & -1.4489 \\
\hline 7.0 & 1 & -14.2599 \\
\hline 0.71 & 2 & -2.1572 \\
\hline 0.71 & 3 & -2.8667 \\
\hline
\end{tabular}

Table 3: Rate of mass transfer at the plate

\begin{tabular}{|c|c|c|c|}
\hline $\boldsymbol{S}_{\boldsymbol{c}}$ & $\boldsymbol{K}_{\boldsymbol{c}}$ & $\boldsymbol{S}_{\boldsymbol{0}}$ & $\boldsymbol{S}_{\boldsymbol{h}}$ \\
\hline 0.22 & 1 & 1 & -0.3552 \\
\hline 0.30 & 1 & 1 & -0.4038 \\
\hline 0.60 & 1 & 1 & -0.5407 \\
\hline 0.22 & 2 & 1 & -0.5690 \\
\hline 0.22 & 3 & 1 & -0.7315 \\
\hline 0.22 & 1 & 2 & -0.1134 \\
\hline 0.22 & 1 & 3 & 0.1284 \\
\hline
\end{tabular}

\section{Conclusions}

Analytical solutions are obtained for the unsteady flow past a vertical porous plate in slip flow regime in the presence of heat source, thermal diffusion, and chemical reaction. The dimensionless governing equations are solved by using perturbation technique. The conclusions are as follows:

- The velocity profiles enhance for increasing the $h, G_{T}, G_{M}$ and $K$.

- The temperature profiles decrease with increasing $H$.

- Prandtl number strongly influences the temperature profiles.

- The concentration profiles increase with increasing $S_{0}$ while the trend reversed for $K_{c}$ and $S_{c}$.

- The results would be useful in many areas related to the diffusive operations which involve the molecular diffusion of species with chemical reaction. 


\section{Acknowledgments}

The authors are thankful to the reviewers for their suggestions that significantly improved our paper.

\section{References}

Alam, M.S.,and Rahman, M. M. (2005):Dufour and Soret effects on MHD free convective heat and mass transfer flow past a vertical porous plate embedded in a porous medium. Journal of Naval Architecture and Marine Engineering. Vol. 10, no. 3, pp. 1-8.

Anjalidevi, S. P. and Kandasamy, R. (2000): Effects of chemical reaction heat and mass transfer on MHD flow Alam, M. M. and Islam, N. (2007):Dufour and Soret effects on steady MHD free convection and mass transfer fluid flow through a porous medium in a rotating system. Journal of Naval Architecture and Marine Engineering 4, pp. 43-55. .http://dx.doi.org/10.3329/jname.v4i1.915

past a semi-infinite plate,Z.Angew. Math. Mech., vol. 80, pp. 697-700.

http://dx.doi.org/10.1002/1521-4001(200010)80:10<697::aid-zamm697>3.0.c0;2-f

Chambre, P. L. and Young, J. D., (1958): on the diffusion of a chemically reactive species in a laminar boundary layer flow, Physics of Fluids, vol.1, pp.48 -54.

http://dx.doi.org/10.1063/1.1724336

Das, U. N., Deka, R.K. and Soundalgekar, V. M. (1994): Effects of mass transfer on flow past an impulsively started infinite vertical plate with constant heat flux and chemical reaction, Forschung in ingenieurwesen, vol. 60, pp. 284-287. http://dx.doi.org/10.1007/BF02601318

Das, S. S. and Mitra, M.(2009): Unsteady mixed convective MHD flow and mass transfer past an accelerated infinite vertical plate with suction, Indian Journal of Science and Technology, Vol. 2, No. 5, pp. 18 - 22.

Das, S. S., Sahoo, S. K. and Dash, G. C. (2006): Numerical solution of mass transfer effects on an unsteady flow past an accelerated vertical porous plate with suction, Bull, Malays.Math. Sci. Soc., Vol. 29, No. 1, pp. 33 - 42.

Das, S. S., Satapathy, A., Das, J. K. and Sahoo, S. K. (2007): Numerical solution of unsteady free convective MHD flow past an accelerated vertical plate with suction and heat flux, J. Ultra Sci. Phys. Sci., Vol. 19, No. 1, pp. $105-112$.

Das, S. S., Satapathy, A., Das, J. K. and Panda, J. P. (2009): Mass transfer effects on MHD flow and heat transfer past a vertical porous plate through a porous medium under oscillatory suction and heat source, Int. J. Heat Mass Transfer, Vol. 52, pp. 5962 - 5969.

http://dx.doi.org/ 10.1016/j.ijheatmasstransfer.2009.04.038

Kumar, J. G., Satyanarayana, P. V. and Ramakrishna, S. (2009): Effects of chemical reaction and mass transfer on MHD unsteady free convection flow past an infinite vertical plate with constant suction and heat sink, J.Ultra Scientist, Vol. 21, No. 3, pp. $12-28$.

Hayat, T., Mustafa, M. and Pop, I., (2010): Heat and mass transfer for Soret and Dufour effects on mixed convection boundary layer flow over a stretching vertical surface in a porous medium filled with a visco elastic fluid, Communications in Nonlinear Science and Numerical Simulation, Vol. 15, No. 5, pp. 1183 - 1196. http://dx.doi.org/10.1016/j.cnsns.2009.05.062

Kumar, B. R. andGangadhar, K.. (2012):Heat generation effects on MHD boundary layer flow of a moving vertical plate with suction, Journal of Naval Architecture and Marine Engineering, vol. 9, no. 2, pp. 153-167. http://dx.doi.org/10.3329/jname.v9i2.8550

Kumar, B. R. (2013): MHD boundary layer flow on heat and mass transfer over a stretching sheet with slip effect, Journal of Naval Architecture and Marine Engineering,Vol.10, No2, pp.16-26.

http://dx.doi.org/10.3329/jname.v10i2.16400

Kumar, B. R., Kumar, T. S. and Kumar, A. G. V. (2015): Thermal diffusion and radiation effects on unsteady free convection flow in the presence of magnetic field fixed relative to the fluid or to the plate, Frontiers in Heat and Mass Transfer (FHMT), vol. 6, no. 1.

http://dx.doi.org/10.5098/hmt.6.12

Mishra, B. K. (2005): Heat transfer in MHD free convection flow over an infinite vertical plate with timedependent suction,ActaCienciaIndica, XXXI M No.2, pp.371. 
Muthucumaraswamy, R and Ganesan, P (2001): First order chemical reaction on flow past an impulsively started vertical plate with uniform heat and mass flux, Acta Mechanica, vol. 147, pp. 4557.http://dx.doi.org/10.1007/BF01182351

Pal, D. and Talukdar, B. (2010): Perturbation analysis of unsteady Magnetohydrodynamic convective heat and mass transfer in a boundary layer slip flow past a vertical permeable plate with thermal radiation and chemical reaction, Communications in Nonlinear Science and Numerical Simulation, vol. 15, pp. 1813-1830. http://dx.doi.org/10.1016/j.cnsns.2009.07.011

Poonia, H. and Chaudhary, R. C. (2010): MHD free convection and mass transfer flow over an infinite vertical porous plate with viscous dissipation, Theoret. Appl. Mech., Vol. 37, No. 4, pp. 263 - 287.

http://dx.doi.org/10.1063/1.1724336

Rao, B. M., Reddy, B.G. V., Raju, M. C. and Varma, S.V.K. (2013): MHD transient free convection and chemically reactive flow past a porous vertical plate with radiation and temperature gradient dependent heat source in slip flow regime, IOSR Journal of Applied Physics. Volume 3, Issue 6, pp. 2232.http://dx.doi.org/10.9790/4961-0362232

Saxena,S. S. and Dubey,G. K. (2009): Unsteady MHD heat and mass transfer free convection flow of polar fluids past a vertical moving porous plate in a porous medium with heat generation and thermal diffusion, Advances in Applied Science Research, vol. 2, no. 4, pp.259-278.

Sharma, P. R. and Mathur, P. (1995): Steady laminar free convection flow of an electrically conducting fluid along a porous hot vertical plate in the presence of heat source/sink. Indian J. Pure Appl. Math., vol. 26, no.11, pp. 1125-1134.

Sharma P. R. and Singh, G. (2008): Unsteady MHD free convective flow and heat transfer along a vertical porous plate with variable suction and internal heat generation. Int. J. of.Appl. Math and Mech., vol. 4, no. 5, pp.1-8.

Sharma, P. K. and Chaudary, R. C. (2003): Effect of variable suction on transient free convection viscous incompressible flow past a vertical plate with periodic temperature variations in slip-flow regime, Emirates J. Engineering Research, Vol. 8, pp. 33-38.

Sharma, P. K. (2005): Fluctuating thermal and mass diffusion on unsteady free convective flow past a vertical plate in slip-flow regime, Latin American Applied Research, Vol.35, pp. 313-319.

Singh, N. P., Kumar, A., Singh A. K. and Singh, Atul K.(2006): MHD free convection flow of viscous fluid past a porous vertical plate through non homogeneous porous medium with radiation and temperature gradient dependent heat source in slip flow regime, Ultra Science, vol.18, no. 1, M, pp.39-46.

Varshney, N. K. and Kumar, S. (2004): Effect of thermal diffusion on steady laminar free convective flow along a moving porous hot vertical plate in the presence of heat source with mass transfer, Acta Ciencia Indica, XXX M No.2, pp.489-495. 


\section{APPENDIX}

The constants involved in the solution of the problem are:

$$
\begin{aligned}
& m_{1}=p_{r}(1+H) \\
& m_{3}=\frac{S_{c}+\sqrt{S_{c}+4 K_{c}} S_{c}}{2} \\
& m_{5}=\frac{1+\sqrt{1+4 M_{1}}}{2}
\end{aligned}
$$$$
A_{1}=\frac{p_{r} m_{1}}{m_{1}^{2}-\left(p_{r}+p_{r} H\right) m_{1}+\frac{n p_{r}}{4}}
$$$$
A_{3}=\frac{S_{c} S_{0} A_{1} m_{1}^{2}+S_{c} A_{2} m_{1}}{m_{1}^{2}-S_{c} m_{1}-S_{c}\left(\frac{n}{4}-K_{c}\right)}
$$$$
A_{5}=\frac{S_{c}\left(1-A_{2}\right) m_{3}}{m_{3}^{2}-S_{c} m_{3}+S_{c}\left(\frac{n}{4}-K_{c}\right)}
$$$$
A_{7}=\frac{-G_{M}\left(1-A_{2}\right)}{m_{3}^{2}-m_{3}-M_{1}}
$$$$
A_{9}=\frac{-G_{T} A_{1}-G_{M} A_{3}+A_{6}}{m_{1}^{2}-m_{1}+M_{2}}
$$$$
A_{11}=\frac{A_{7} m_{3}-G_{M} A_{5}}{m_{3}^{2}-m_{3}+M_{2}}
$$$$
A_{13}=\frac{A_{8} m_{5}}{m_{5}^{2}-m_{5}+M_{2}}
$$$$
A_{14}=\frac{-A_{9}-A_{10}-A_{11}-A_{12}-A_{13}-h m_{1} A_{9}-h m_{2} A_{10}-h m_{3} A_{11}-h m_{4} A_{12}-h m_{5} A_{13}}{\left(1+h m_{6}\right)}
$$$$
A_{15}=-m_{1} A_{6}-m_{3} A_{7}-m_{5} A_{8}
$$$$
A_{16}=-m_{1} A_{9}-m_{2} A_{10}-m_{3} A_{11}-m_{4} A_{12}-m_{5} A_{13}-m_{6} A_{14}
$$$$
A_{17}=A_{1}\left(m_{2}-m_{1}\right)
$$$$
A_{18}=-m_{3}+A_{2}\left(m_{3}-m_{1}\right)
$$$$
m_{4}=\frac{S_{c}+\sqrt{S_{c}^{2}-S_{c}\left(n-4 K_{c}\right)}}{2}
$$$$
m_{6}=\frac{1+\sqrt{1-4 M_{2}}}{2}
$$

$$
A_{2}=\frac{-S_{c} S_{0} m_{1}^{2}}{m_{1}^{2}-S_{c} m_{1}-K_{c} S_{c}}
$$

$$
A_{4}=\frac{-S_{c} S_{0} A_{1} m_{2}^{2}}{m_{2}^{2}-S_{c} m_{2}+S_{c}\left(\frac{n}{4}-K_{c}\right)}
$$

$$
A_{6}=\frac{-G_{T}-G_{M} A_{2}}{m_{1}^{2}-m_{1}-M_{1}}
$$

$$
A_{8}=\frac{-A_{6}\left(1+h m_{1}\right)-A_{7}\left(1+h m_{3}\right)}{\left(1+h m_{5}\right)}
$$

$$
A_{10}=\frac{G_{T} A_{1}-G_{M} A_{4}}{m_{2}^{2}-m_{2}+M_{2}}
$$$$
A_{12}=\frac{G_{M}\left(A_{3}+A_{4}+A_{5}\right)}{m_{4}^{2}-m_{4}+M_{2}}
$$

$$
m_{2}=\frac{\left(p_{r}+p_{r} H\right)+\sqrt{\left(p_{r}+p_{r} H\right)^{2}-n p_{r}}}{2}
$$

$$
A_{19}=m_{4}\left(A_{3}+A_{4}+A_{5}\right)-m_{1} A_{3}-m_{2} A_{4}-m_{3} A_{5}
$$

